\title{
ESTÍMULOS QUE FAVORECEM O TREINAMENTO NO LABORATÓ RIO DE ENFERMAGEM: REVISĀO DE LITERATURA
}

\author{
Maria Romana Friedlander* \\ Cristina Seika Tanaka** \\ Paula Sue F. de Siqueira**
}

FRIEDLANDER, M. R.; TANAKA, C. S.; SIQUEIRA, P. S. de. Estímulos que favorecem o treinamento no laboratório de enfermagem: revisão de literatura. Rev.Esc.Enf.USP, São Paulo, 23(2): - , ago. 1989.

Foi realizada uma revisão da literatura relacionada à motivação da aprendizagem, especificamente dos aspectos relativos às habilidades manuais e a outros de interesse para o treinamento de estudantes de graduaçāo no laboratório de enfermagem. Conclui que a literatura indica algumas condiçôes que podem estimular ou desestimular o treinamento das habilidades motoras como atividade de livre opção dos estudantes. Essas condiçōes estāo relacionadas ao professor, aos procedimentos ou técnicas, ao laboratorio, ao sistema de avaliação e ao proprio aluno.

Há vários anos os estudantes do curso de enfermagem têm corno opção de aprendizagem o treinamento das técnicas básicas no laboratório de enfermagem (LE) sem a obrigatoriedade de cumprimento de horário ou da presença do professor. Essa atividade já foi descrita em trabalho anterior (FRIEDLANDER et alii 1984) c continua a ser observada e estudada tendo em vista a constatação que o treinamento previo de estudantes no LE diminui o número de erros cometidos na primeira experiência em cliente (FRIEDLANDER, 1984) e que o LE pode tornar-se um recurso instrucional de grande utilidade no ensino (FRIEDLANDER, 1986).

Analisando-se os dados de 1985, 1986 e 1987 observou-se que uma das turmas frequentou o laboratório com certa assiduidade enquanto as demais pouco compareceram para treinar qualquer técnica. Em 1985, os 67 estudantes que terminaram a disciplina Fundamentos de Enfermagem 1 foram ao laboratório 526 vezes, ou seja, 7,8 vezes em média por aluno. Em 1986 e 1987, respectivamente, a média por aluno foi de 2,9 e 3,2, ou seja, menos da metade que em 1985.

UNITERMOS: Ensino de Enfermagem, treinamento em laboratório.

* Enfermeira. Professor Assistente Doutor do Departamento de Enfermagem Médico-Cirúrgica da Escola de Enfermagem da Universidade de São Paulo.

** Alunas do 3* ano de Enfermagem, monitoras da disciplina Fundamentos de Enfermagem I da Escola de Enfermagem da Universidade de São Paulo. 
Levantou-se, assim, a hipótese de que deveria existir algum fator ou condição estimulante (em 1985) ou desestimulante (em 1986 e 1987) que pudesse explicar as diferenças marcantes no comportamento das três turmas de estudantes uma vez que as condiçōes de ensino mantiveram-se relativamente semelhantes.

A fim de compreender melhor as causas das diferenças de comportamento dos grupos de estudantes decidiu-se fazer uma revisão da literatura ligada à motivação da aprendizagem, especificamente aqueles aspectos relacionados às habilidades manuais ou a outros de interesse para o treinamento no laboratório de enfermagem.

Todo o professor pretende, em princípio, desenvolver um trabalho com sucesso e preocupa-se frequentemente com a falta de resposta positiva dos estudantes aos seus esforços didáticos. Compreende que não pode simplesmente culpar o estudante por falta de atenção ou interesse e começa a questionar o problema dos motivos que levam alguns estudantes a responderem bem e outros a não se sentirem atraídos pelas suas iniciativas. A motivação torna-se, então, uma preocupação para o docente bem como para todo o profissional que trabalhe com alguma forma de comportamento humano. Acredita-se que, para acompanhar o desenvolvimento do futuro e fazer frente aos inúmeros problemas mundiais, a formação do ser humano precisa ser revista e uma das questōes básicas é como motivar os jovens. SPEECE, (1986) sugere, entre outras coisas, que os professores de ciências demonstrem um real entusiasmo pelo que estão fazendo.

Portanto, a revisão de literatura proposta pode auxiliar o docente de enfermagem, de todos os níveis de ensino, na sua tarefa pedagógica e facilitar, aos profissionais dos setores de educação continuada, o desempenho de sua função de treinar e reciclar o pessoal de serviço.

A importância da motivação para a aprendizagem ter evoluído e se expandido muito nas últimas décadas com as novas concepçōes do processo ensino-aprendizagem passando a constituir hoje "o centro de interesse de todo o processo educativo" (CAMPOS, 1984) e a "desempenhar um papel muito importante nas teorias da aprendizagem...", KLAUSMEIER, (1977) e WITTER (1984) afirmam que apesar de muito se ter pesquisado sobre o assunto e do fato da motivaçāo ser um problema central da psicologia, ainda há muitos aspectos com falta de consenso e discussōes em torno de muitas questōes. LARCEBEAU (1983) numa revisão teórica da motivação e da personalidade, sob o ponto de vista psicológico, verifica que para alguns autores motivação é a própria fonte de personalidade.

Motivação é um processo que ocorre no interior do indivíduo e que o impulsiona para desenvolver uma atividade, um comportamento ou uma ação com a finalidade de atingir um objetivo determinado. Motivo, portanto, é aquilo que motiva, que impele o indivíduo a comportar-se de determinada maneira ou que provoca a motivação (AUSUBEL, 1980; CAMPOS, 1984; NÉRICI, 1984). Apesar de ser 
um assunto de extrema relevância, a motivação não é inprescindível à aprendizagem. Aprendem-se muitas coisas sem se estar motivado (AUSUBEL, 1980).

Chama-se estimulaçāo, o processo de despertar uma reaçāo no indivíduo, sendo, contudo, alheia ou externa à própria reaçāo. $O$ estímulo apenas deflagra a reação, e o papel do professor é manipular estímulos que possam facilitar a aprendizagem do estudante (CAMPOS, 1984; NERICI, 1984). RASCHKE (1986) apresenta um exemplo e RASCHKE et alii (1987) defendem os sistemas de estímulos criados para estudantes com dificuldades de aprendizagem.

A explicaçāo da dinâmica da motivação e dos mecanismos intrínsecos do comportamento humano é área de estudo da psicologia e a motivação é compreendida dentro de enfoques diferentes dependendo da teoria que a fundamenta. GOEBEL \& BROWN (1981) explicam que as teorias da motivação inicialmente estavam relacionadas com situações específicas e níveis de motivos específicos mas, com o desenvolvimento da psicologia, há uma tendência em ver os motivos como uma constante da vida.

Sob o ponto de vista da teoria da aprendizagem associacionista, defendida por Thordike, Hull, Skinner e outros, a tendência para um ser responder em determinada direção ou em um certo ritmo é resultante de um estado de privação ou de satistação e da qualidade do reforço que recebe. Desta forma a intensidade ou o sentido da resposta é determinado pelo esquema de reforçamento (CORIA-SABINI, 1986). Assim a motịvação iniciar-se-ia com uma necessidade real ou em potencial produzindo impulsos que levam o indivíduo a desenvolver comportamentos específicos. A reduçāo da tensāo ocasionada pela satisfação da necessidade seria o reforçamento que, por sua vez, asseguraria a aprendizagem de um determinado comportamento. Assim, para estes teóricos comportamentalistas, o comportamento do estudante pode ser modelado pelos professores até que ele próprio se auto-reforce.

Esses psicólogos estudaram o fenômeno da motivação com base em experiências em animais e nas necessidades biológicas, mas a definem da mesma forma para os seres humanos e para todas as demais necessidades.

Os estudiosos da linha cognitivista, no que se refere à aprendizagem, valorizam os motivos intrínsecos característicos dos seres humanos e que não dependem de reforço externo. Dão mais atenção que os da linha anterior aos objetivos, intenções, expectativas e planos pessoais do próprio indivíduo, dos quais os seres humanos têm consciência de que controlarão a direção da atividade. Neste caso o papel do professor nāo seria reforçar o comportamento desejado, mas proporcionar condiçōes para que esses motivos intrínsecos ao indivíduo fossem atendidos. AUSUBEL (1980) afirma que a motivação de realizaçāo consiste em proporções variadas de impulso cognitivo (vontade de aprender), impulso afiliativo (necessidade de aipruvação dos pais, protessores. coleọas e outros) r: motivação de engrandeci- 
mento do ego (auto-afirmação e auto-estima) dependendo de várias características da personalidade, de determinantes genéticos, determinantes sócio-culturais e interação social.

Dentro das teorias de personalidade da linha humanista encontramos, como principais representantes, A. H. Maslow e C. R. Rogers.

Para Rogers o processo de mudança dirige-se para a auto-realização, a maturidade e o auto-governo. O professor só pode confiar no estudante porque lhe é inerente o desejo de maturação e de realização mas não poderia pensar em motiválo. Seu papel seria o de facilitador cujos conhecimentos e experiência apenas seriam tomados como um recurso ao alcance do estudante.

Maslow definiu o comportamento como produto da satisfação ou da privação do atendimento das necessidades humanas hierarquicamente estabelecidas. O que uma pessoa pode fazer é não prover os fatores de atendimento e, com isso, aumentar o nivel de tensão da necessidade e reabixar a satisfaçāo. Ou, por outro lado, pode prover aquilo que satisfaz uma determinada necessidade e diminuir essa tensāo. As necessidades são próprias da natureza específica de uma pessoa, intrínseca a ela e estritamente individuais. ARCHER (1978) explica que um indivíduo não poderia motivar o outro. Os fatores de satisfação ou contra-satisfação podem, no entanto, modificar a direção do comportamento porque estimulam a menor ou maior necessidade motivadora. Compreende-se, por essa abordagem, que um fator, uma condiçāo ou um esforço desenvolvido pelo professor pode estimular um aluno e nāo produzir efeito em outro. A hierarquia das necessidades básicas de Maslow representa uma tentativa para explicar a dinâmica dos princípios motivacionais que inter-relicionam os episódios comportamentais durante todo o desenvolvimento do ser humano e em todas as culturas (GOEBEL \& BROWN, 1981).

Roger e Maslow não testaram suas teorias em laboratórios mas têm obtido sucessos significativos no campo clínico, junto a estudantes universitários e no campo social.

A psicologia educacional, que muito tem contribuído para a compreensão e o conhecimento da riiotivação, preocupa-se essencialmente com a aprendizagem do comportamento humano seja qual for a situação. Contudo, sob o ponto de vista da didática, em que a aprendizagem visada é aquela que se dá em situações formais, em escolas, com programas determinados e espaços de tempo restritos, há uma preocupação profunda com os estímulos e as condiçōes externas que contribuem para o sucesso escolar do estudante. Neste caso precisa-se lidar com variáveis relacionadas ao professor, às condiçōes do ambiente escolar, às estratégias e métodos de ensino, características dos programas e atividades que compöem o currículo escolar.

Assim, é frequente, pelos professores, o uso do termo motivaçāo com a conotação extrínseca, como sinônimo de estímulo e incentivo, com isto significar 118

Reve. Ess. Einf. LiSP, Săo Baulo, 23(2):115-125, abr. 1989 
que não é considerada a motivação intrínseca. NÉRICI (1984) define motivação como "o processo de incentivo destinado a desencadear impulsos no interior do indivíduo a fim de predispô-lo a querer participar das atividades escolares oferecidas pelo professor". Acrescenta que não há como a aprendizagem sem esforço por parte do estudante, o professor precisa poder e saber utilizar certos recursos que deflagram a vontade de aprender. SLAVIN (1984) destingue "motivação do estudante" e "incentivo em classe". Usa o primeiro termo para se referir ao interesse do estudante pelos trabalhos acadêmicos e a aprendizagen e o segundo ao se referir aos métodos que os professores usam para despertar o interesse do estudante para a atividade acadêmica (sistema de notas, prêmios, recompensas, recebimento das respostas corretas, etc).

A concepçāo pedagógica clássica classifica a motivação em motivação intrínseca (relacionada ao objeto da aprendizagem e derivada da satisfação pela própria aprendizagem) e a motivação extrínseca (não resultante do interesse pela matéria em si mas de fatores externos ao conteúdo a ser aprendido) e aponta dois tipos de efeitos, o positivo e o negativo. $O$ positivo nāo resulta em prejuízos psicolúgicos para o aluno e tende a manter-se mais estável, duradouro e leva a uma aprendizagem realmente profunda. O efeito negativo é aquele que, apesar de conduzir à aprendizagem, pode tornar o estudante inseguro, tímido ou violento (CAMPOS, 1984; NÉRICI, 1984; WITTER \& LOMÔNACO, 1984).

A pesquisa tem apoiado com bastante clareza que há uma relaçāo entre o estabelecimento de objetivos específicos e o desempenho do ser humano tanto na área da educação quanto na do trabalho industrial (HALL et alii, 1987).

LEE \& EDWARDS (1984) afirmam que, em relação ao estabelecimento de objetivos na área das habilidades motoras e o efeito disso no desempenho dos estudantes, a pesquisa tem mostrado extensivamente que um estudante suficientemente motivado pelo estabelecimento bem definido de objetivos apresenta um aumento significativo no nível de desempenho quando comparado a estudantes com objetivos nāo específicos. Numa revisāo de 110 trabalhos de pesquisa, Locke, Seari, Shaw e Lathan citados por PASCARELLA et alii (1981) verificam que 90\% dos estudos apoiam esta afirmação.

SALILI et alii (1976), interessados em verificar se as recompensas extrínsecas ou as condições de avaliaçāo podem reduzir a motivação continuada ou o interesse intrínseco contínuo comparando o desempenho de crianças (de 10 a 12 anos) frente a três condiçōes de avaliaçāo (nota do professor, auto-avaliação e comparação com colegas) verificaram que a avaliação externa, principalmente a realizada por nota do professor é um instrumento potencialmente perigoso para a manutençāo da motivação contínua ou a manutenção do interesse contínuo em relaçāo ao processo de aprendizagem. A avaliação externa parece reduzir a percepçāo do estudante quanto à responsabilidade no desempenho de uma tarefa. 
Ainda $\mathrm{cm}$ relação ao efeito da definiçāo de objetivos e recompensas no desempenho de estudantes, SCHUNK (1984) verificou que ambos os fatores juntos produzem melhores resitados que cada um deles em separado.

Ao se tratar de pessoas no final da adolescência ou adultas os motivos precisam estar ligados às características individuais, sócio-econômicas, interesses, valores, ideais, preferências e propósitos de cada aprendiz, (CAMPOS, 1984) uma vez que estes trazem consigo uma experiência e vivência anteriores que lhes reforçam suas próprias características. AUSUBEL (1980) afirma que, dentro de limites razoáveis, a puniçāo (não recompensa ou medo do fracasso) exerce influência na educação sistemática, particularmente no ensino universitário e profissional devido à tendência humana à procrastinação. Chama a atenção para a importância, no adulto, dos símbolos de status e das fontes de auto-estima.

LEIGHBODY \& KIDD (1977) auxiliam o assunto quando aconselham os professores a desenvolver algumas iniciativas para manter a atençāo do estudante durante os trabalhos escolares, despertar o interesse do aluno, manter a vontade de aprender e considerar as diferenças no que concerne à aprendizagem entre os estudantes adultos e os mais jovens. SLAVIN (1984) defende a "aprendizagem cooperativa" alegando que quando o benefício da recompensa recai sobre um grupo, é mais valorizado pelos estudantes que quando o mesmo benefício reverte somente para um indivíduo.

MAGER (1983) acredita na influência profunda que o professor exerce na atitude mais ou menos favorável do estudante em relação a um assunto ou matéria. Explana detalhadamente sobre os fatores que condicionam a atitude dos estudantes e sugere uma série de medidas ao alcance do professor que poderão melhorar o resultado da aprendizagem escolar. Deixa claro que a atitude favorável do professor estimula a atitude positiva do estudante em relação à sua disciplina. Aconselha também o docente a clarificar os objetivos, identificar os meios que auxiliarão a alcançar esses objetivos, explorar os procedimentos úteis para testar o ensino e verificar as ocorrências impeditivas.

$\mathrm{Na}$ área da enfermagem, que também tem dedicado alguns dos seus esforços a tentar respostas para muitas questões relacionadas à aprendizagem, não se tomou conhecimento de trabalhos relacionados diretamente à motivaçāo no ensino das habilidades psicomotoras.

Contudo, SATO (1985) relata um estudo de caso relacionado à motivação no treinamento clínico de enfermagem e a importância da atitude do professor. Enfatiza que o professor deve auxiliar para que o interesse interno do aprendiz se transforme em desenvolvimento dependendo do papel do professor na motivaçāo para a aprendizagem.

TORNYAY (1984) faz uma revisāo da pesquisa relacionada ao processo ensino-aprendizagem em enfermagem de 1971 a 1982. Verifica que valores e atitu- 
des do professor influenciam o desempenho dos estudantes principalmente na área clínica. Não relata nenhum estudo específico da motivação para o desempenho de habilidades motoras.

NORONHA (1985) fez uma revisāo de literatura sobre motivação, sua conceituação, o papel da mesma na prestação da assistência profissional e no processo ensiro-aprendizagem na enfermagem. Destaca como fator de motivação a importância das atividades práticas dos estudantes tanto na assistência como no planejamento de uma disciplina.

NOCA et alii (1985), descrevendo um estudo das características do treinamento de estudantes no LE verificou que, em 95 respostas de estudantes, $38 \%$ afirmaram frequentar o laboratório motivados pela auto-percepção da falta de habilidade, $26 \%$ por medo de enfrentar a situação real sem a devida segurança e 23\% afirmaram ser estimulados pelo docente.

Como conclusão do estudo da literatura relatada acredita-se que se pode indicar algumas condiçōes que estimulam ou desestimulam o estudante a treinar as habilidades psicomotoras no LE como atividade de livre opção. Essas condições são produto de uma revisão teórica e precisariam ser testadas experimentalmente, em trabalhos posteriores para que sua eficiência fosse ou nāo comprovada frente às nossas condiçōes particulares de ensino.

\section{A - Condiçöes que podem estimular}

a) Relacionadas ao professor:

1. O professor mostra de forma clara e precisa os objetivos do treinamento de cada uma das técnicas.

2. O professor demonstra entusiasmo pelo treinamento dos alunos no LE.

3. $\mathrm{O}$ professor elogia com frequência as habilidades bem treinadas.

4. O professor mostra explicitamente ao estudante aquelas habilidades que ele não executa bem e que deveriam ser melhor treinadas.

5. O professor está disponível quando o estudante precisa de auxllio para treinar no LE.

6. O professor de estágio no campo é exigente e cobra a habilidade.

b) Relacionadas às técnicas em si:

1. O campo de estáłio é muito rico em oportunidades de execução de técnicas.

2. A técnica parece complexa durante a aula teórica ou a demonstração.

3. A técnica já é conhecida do aluno (viu alguém fazer ou ouviu alguém mencioná-la) antes de começar a disciplina Fundamentos de Enfermagem. 
4. A técnica já foi necessária ao aluno (para atender alguém da familia ou colega) que não a executou por não conhecê-la.

c) Relacionadas ao Laboratório de Enfermagem:

1. O laboratório conta com espaço físico, claridade e boa disposição do equipamento.

2. O laboratório conta com a presença do aluno-monitor.

3. O laboratório conta com a presença do professor.

4. O laboratório conta com material e equipamento suficientes em quantidade e qualidade.

5. O laboratório encontra-se aberto em horário conveniente.

6. O laboratório conta com várias formas de treinamento (treinamento em manequins, em colegas, roteiros auto-instrucionais e outros).

d) Relacionadas ao sistema de avaliação:

1. Há previsão de provas práticas na disciplina.

2. Há previsão de provas teóricas que podem conter questões práticas na disciplina.

3. O estágio é avaliado por meio de critérios que consideram as habilidades manuais.

e) Relacionadas ao aluno:

1. O aluno tem medo de se sentir inseguro junto ao cliente.

2. O aluno quer ser um profissional reconhecido por seus pacientes, por seus colegas e por outros profissionais.

3. O aluno quer ser respeitado em seu ambiente escolar.

4. O aluno quer obter sucesso nos estudos e ser considerado pela familia, professores, amigos e colegas.

5. O aluno quer saber bem as técnicas porque gosta das habilidades manuais.

6. O aluno é uma pessoa curiosa.

7. O aluno quer formar-se o mais rápido possível para trabalhar e ganhar o próprio sustento.

8. O aluno quer ficar livre de compromisso relacionado à aprendizagem das técnicas para poder realizar outras atividades.

B - Condições que podem desestimular:

a) Relacionadas ao pro@essor: 
1. O professor nāo define os objetivos do treinamento de cada uma das técnicas com clareza.

2. O professor pouco se refere ao treinamento no LE como recurso de aprendizagem.

3. O professor não valoriza as técnicas que o aluno treinou.

4. O professor não tem disponibilidade quando o aluno lhe pede auxilio para treinar no LE.

5. O professor salienta a facilidade com que a técnica pode ser executada sem treinamento específico.

6. O professor de estágio no campo nāo exige muito em relaçāo à perfeiçāo da execução das técnicas.

7. O professor ou monitor transmitem muita ansiedade ao estudante durante o treinamento no LE.

ذ) Relacionadas às técnicas em si:

1. As técnicas parecem muito fáceis ao serem demonstradas.

2. As técnicas parecem pouco úteis (ou de uso pouco comum ou de uso remoto) durante a demonstraçāo ou a aula teórica.

3. O campo de estágio usado pela disciplina é pobre em número de oportunidades para a execuçāo das técnicas.

c) Relacionadas ao laboratório de enfermagem:

1. O laboratório conta com pouco espaço físico, pouca claridade ou com uma disposição do material pouco conveniente.

2. O lahoratório não conta com a presença do monitor.

3. O laboratório năo conta com a presença de um professor.

4. O laboratório nāo se encontra aberto em horário conveniente.

5. O laboratório não conta com equipamento e material suficiente em quantidade e qualidade.

d) Relacionadas ao sistema de avaliação:

1. Não há previsão de provas práticas na disciplina.

2. Não há critérios para avaliação de estágio relacionados à habilidade manual.

e) Relacionadas ao aluno:

1. O aluno é muito nervoso.

2. O aluno já tem experiência anterior na execuçāo das técnicas. 
3. O aluno tem dificuldade excessiva na execução de atividades manuais.

4. O aluno já teve experiências desagradáveis com um treinamento anterior semelhante.

5. O aluno tem pouca necessidade pessoal de auto-realização.

FRIEDLANDER, M.R.; TANAKA, C.S.; SIQUEIRA, P.S.F. de Conditions that encourage laboratory training: a literature review. Rev. Esc. Enf. USP, São Paulo, 23(2): - Aug. 1989.

This article is a review of literature related to what motivates nursing graduates to attend freely a laboratory to better develop practical skills. It concluded that the literature indicates conditions that stimulate or discourage training of practical skills. These conditions are related to the professor, to techniques or procedures, to laboratory conditions, to evaluating systems and to the student himself.

UNITERMS: Nursing education, laboratory training.

\section{REFERÊNCIAS BIBLIOGRÁFICAS}

ARCHER, E.R. The myth of motivation. The personnel administrator. Dec. 1978.

AUSUBEL, D.P.; NOVAK, J.D.; HANESIAN, H. Psicologia educacional. 2 cd. Rio de Janeito, Interamericana, 1980.

CAMPOS, D.M. de S. Psicologia da aprendizagem. 16 ed. Petropolis, Vozes, 1984.

CORIA-SABINI, M.A. Psicologia aplicada à educação. Sāo Paulo, IP U, 1986.

FRIEDLANDER, M.R. O ensino dos procedimentos băsicos no laboratório de enfermagem. Rev. Esc. Enf. USP, Sāo Paulo, 18(2): 151-62, ago. 1984.

; SCHVARTL, E.; TAVARES, S.R. de A.G.B.; NOCA, C.R. da S. Frequência dos estudantes ao laboratório de enfermagem como atividade de livre opçăo. Rev. Gaúcha Enf., Porto Alegre,5(2) 193-201, jul. 1984.

O laboratório de enfermagem como recurso instrucional. Rev, Paul. Enf., São Paulo, 6(1) 7-9, jan/mar. 1986.

GOEBEL, B.L.; BROWN, D.R. Age differences in motivation related to Maslow's need hierarchy. Dev. Psychol., Washington, 17(6): 809-15. 1981 .

HALL, H.K.; WEINBERG, R.S.; JACKSON, A. Effects of goal specificity, goal difficulty and information feedback on endur ance performance. J. Sport Psychol., Champaign, 9: 43-54. 1987.

K.LAUSMEIER, H.J.; GOODWIN, W. Manual de psicologia educacional: - aprendizagem e capacidade humanas. São Paulo, Harper \& Row do Brasil, 1977.

LARCEbeau, S. Motivation et personnalite. Orient. Sco'. Prof., Paris, 12(3) 215-42, 1983.

LEE, A.M. \& EDWARDS, R.V. Assigned and self-selected goals as deterninants of motor skill performance. Education, 105(1) 87-91, 1984.

LEIGHBODY, G.B. \& KIDD, D.M. Métodos para o ensino profissionalizante. Săo Paulo, EPU, 1977. $251 \mathrm{p}$.

MAGER, R.F. Atitudes favoráveis ao ensino. $3 \mathrm{~cd}$. Porto Alegre, Globo, 1983. $114 \mathrm{p}$.

NÉRICI, I.G. Didática: uma introdução. São Paulo, Atlas. 1984.

NOCA, C.R. da S.; TAVARES, S.R. de A.G.B.; FRIEDLANDER, M.R.; SCHVART\%, E. Características do treinamento de estudantes no laboratório de enfermagem. Rev. Esc. Enf. USP, São Paulo, 19(2): 145-52, ago. 1985. 
NORONHA, R. Motivaçăo no ensino e na assistência de enfermagem. Rev. Bras. Enf., Brasılia, 38(1) 70-5, jan/mar. 1985.

PASCARELLA, E.T.; WALBERG, H.J.; JUNKER, L.K.; HAERTEL, G.D. Continuing motivation in science for early and late adolescents. Am. Educ. Res. J., Washington, 18(4): 439-52, Winter, 1981.

RASCHKE, D. “Delicious" incentives: a technique to motivate reluctant leamers. Teach. Except. Child, Washington, 19(1): 66-7, Fall 1986.

RASCHKE, D.; DEDRICK, C.; THOMPSON, M. Reluctant learners: innovative contingency packages. Teach. Except. Child., Washington, 19(2): 18-21, Winter 1987.

SALILI, F.; MAEHR, M.L.; SORENSEN, R.L.; FYANS JR., L.J. A further consideration of the effects of evaluation on motivation. Am. Educ. Res. J., Washington, 13(2): 85-102, Spring 1976.

SATO, Y. Motivation in clinical nursing training: significance of ideals of instructors. Kango Kyoiku, Tokyo, 26(6): 337-41, Jun. 1985.

SCHUNK, D.H. Enhancing self-efficacy and achievement through rewards and goals: motivational and informational effects. J. Educ. Res., Bloomington, 78(1): 29-34, Sep/Oct. 1984.

SLAVIN, R.E. Students motivating students to excel: cooperative, incentives, cooperative tasks and student achievement. Elem. School J., Chicago, 85(1): 53-63. 1984.

SPEECE, S.P. Teaching in the year 2000. Sci. Teach. 54-7, Sept. 1986.

TORNYAY, R. de, Research on the teaching-learning process in nursing education. Annu. Rev. Nurs. Res., New York, 2: 193-210, 1984.

WITTER, G.P.\& LOMÔNACO, J.F.B. Psicologia da aprendizagem. Såo Paulo, EPU, 1984.

Recebido para publicaçăo em $02 / 05 / 88$. 